\title{
Solitary Rossby Waves in the Lower Tropical Troposphere
}

\author{
Andre Lenouo ${ }^{1}$ and Francois Kamga Nkankam ${ }^{2}$ \\ ${ }^{1}$ Department of Physics, Faculty of Science, University of Douala, P.O. Box 24157, Douala, Cameroon \\ ${ }^{2}$ Laboratory for Environmental Modelling and Atmospheric Physics, Department of Physics, University of Yaounde 1, \\ Yaoundé, Cameroon
}

Correspondence should be addressed to Andre Lenouo; lenouo@yahoo.fr

Received 27 February 2013; Accepted 8 May 2013

Academic Editors: L. Ahrens, M. N. Lorenzo, and R. Singh

Copyright (c) 2013 A. Lenouo and F. K. Nkankam. This is an open access article distributed under the Creative Commons Attribution License, which permits unrestricted use, distribution, and reproduction in any medium, provided the original work is properly cited.

\begin{abstract}
Weakly nonlinear approximation is used to study the theoretical comportment of large-scale disturbances around the intertropical midtropospheric jet. We show here that the Korteweg de Vries (KdV) theory is appropriated to describe the structure of the streamlines around the African easterly jet (AEJ) region. The introduction of the additional velocity of the soliton $C_{1}$ permits to search the stage where the configuration of the wave structures is going to emerge out of specified initial conditions and this is the direct and inverse cascade method. It was also shown that the configurations of disturbances can be influenced by this parameter so that we can look if the disturbances are in the control or not of their dispersive effects. This permits to explain the evolution of initial conditions of the Tropical Storm (TS) Debby over West Africa from 20 to 24 August 2006.
\end{abstract}

\section{Introduction}

The Rossby waves are the most important in large-scale atmospheric flow processes [1]. For their analysis, it is usually sufficient to study the horizontal structure of waves. Most theories treating the structure of these waves are based on linear models which only take into account their dispersive behaviour. Nonlinear processes are more interesting because they can help to explain, for example, the hurricane spiral bands observed in the tropical zone [2] and energy exchanges between different modes of the waves [3]. Solitary Rossby waves in a zonal flow appear to have been discovered (analytically) by [4] and have been studied subsequently by [5-10]. All invoke Rossby's $\beta$-plane model, in which the northerly gradient of the vertical component of the Earth's rotation is constant.

Many studies have dealt with nonlinear waves and particularly solitary waves in the atmosphere, stating with works by $[11,12]$. On the theoretical level, nonlinear waves have been examined by [3] in the midatmosphere where the African easterly waves (AEWs) are propagated. In the same region, [13] showed that the Korteweg de Vries (KdV) theory is appropriate to describe the Rossby solitary waves. But the physical interpretation of the results in terms of the Rossby solitary waves is not evident and the roles that these waves could influence the structure and energy of these waves have not been examined. Moreover, [14] established that the propagation of the Rossby solitary wave has behaviour closer to those of ridges and troughs. They therefore showed that these waves can travel long distances in the northern hemisphere without a change both in speed or structure, and for any hour.

The first well-known studies of [15] have helped to identify solitary wave connected to internal gravity waves in the atmosphere. They showed that these waves are described by the $\mathrm{KdV}$ equations when they move in the upper atmosphere and by the Benjamin-David-Ono (BDO) equation when they appear in the lower level. They therefore correctly analysed the observations of [16] by using the KdV model, whereas the observation of [17] were better explained by the BDO model. This was the first evidence of these types of wave observed in the atmosphere and their comparison with theoretical models. The solution of the three-dimensional nonlinear Charney-Obukhov equation describing solitary pancake Rossby vortices was found by [18]. Its solution was 
represented in the form of an axially symmetric cylindrical monopole (anticyclonic) vortical structure moving with constant velocity. However, the role of westward-travelling planetary (Rossby) waves in the block onset and the deformation of eddies during the interaction between synopticscale eddies and an incipient block was examined by [10] This author has constructed an incipient block that consists of a stationary dipole wave for zonal wavenumber and a westward-travelling monopole wave with constant amplitude for zonal wavenumber.

The role of nonlinear wave was also being studied in oceanography. Hence, time series observations of nonlinear internal waves in the deep basin of the South China Sea are used to evaluate mechanisms for their generation and evolution by [19]. They showed that internal tides are generated by tidal currents over ridges in the Luzon Strait and steeper as they travel west, subsequently generating highfrequency nonlinear waves. Although nonlinear internal waves appear repeatedly on the western slopes of the South China Sea, their appearance in the deep basin is intermittent and more closely related to the amplitude of the semidiurnal than the predominant diurnal tidal current in the Luzon Strait.

In the present study, we will use the weakly nonlinear theory to examine the behaviour of the large-scale waves around the midtropospheric African easterly jet (AEJ), where the wave is more intensely specified in the case of Tropical Storm (TS) Debby observed over West Africa from 20 to 24 August 2006. Considering that the vertical extent of this jet is smaller than its horizontal extent [3], we admit in first approximation that the motion of the air in this region is dominated by the effects of the rotation of the earth. Under these assumptions, we will look how the nonlinear disturbance could influence the structure and the energy of solitary waves in the midtroposphere where AEWs are propagated. This study is organised as follows. In Section 2, we will present method used to examine the nonlinear vorticity equation. In Section 3, the linear and nonlinear solutions are discussed; a case study of intense AEW of TS Debby is examined whereas conclusion is presented in Section 4 .

\section{Methodology and Data}

2.1. Basics Equations. Rossby solitary waves are sought by using a nonlinear vorticity equation in a barotropic model [13]. This equation integrates the horizontal shear in mean zonal wind. We define a coordinate system $(x, y, t)$, where $t$ is the time component and the space components $x$ and $y$ are along the direction of wave propagation in east and north direction, respectively. When the flow is no divergent, zonal and meridional components of the velocity can be written as a function of a streamfunction perturbation $\psi$ as

$$
u=-\frac{\partial \psi}{\partial y}, \quad v=\frac{\partial \psi}{\partial x}
$$

Otherwise, in a barotropic model, the evolution of streamline is described by the equation [1]

$$
\left[\frac{\partial}{\partial t}+U \frac{\partial}{\partial x}\right] \nabla^{2} \psi+J\left(\psi, \nabla^{2} \psi\right)+\left[\beta-\frac{d^{2} U}{d y^{2}}\right] \frac{\partial \psi}{\partial x}=0
$$

with the following boundary conditions:

$$
\psi=0 \quad \text { at } y=0, \quad y=L .
$$

In (2), $J$ is the Jacobian operator $J(a, b)=(\partial a / \partial x)(\partial b / \partial y)-$ $(\partial a / \partial y)(\partial b / \partial x), \nabla^{2}=\partial^{2} / \partial x^{2}+\partial^{2} / \partial y^{2}$ the Laplacian operator, $U$ the mean zonal wind component, and $\beta$ the meridional gradient of the coriolis parameter.

The effects of nonlinearity are introduced through the Jacobian term, which is nonlinear. In the case of weakamplitude waves, the individual oscillations can be represented in the form of linear or nonlinear wave superposition.

2.2. Theory. A soliton is localised wave, solution to a nonlinear partial derivatives equation without change of velocity or profile in a weakly dispersive area. By using a multiple scale method, we can write a stream function $\psi$ in the form of a power expansion in a small parameter $\epsilon$ so that

$$
\psi=\epsilon \psi_{1}+\epsilon^{2} \psi_{2}+\cdots
$$

It is necessary to introduce a convenient space and time variables $\zeta$ and $\tau$, adapted to describe a weakly dispersive nonlinear system [15]. In this new Galilean reference frame, the transformations of [15] are given by

$$
\zeta=\epsilon^{1 / 2}\left(x-C_{0} t\right), \quad \tau=\epsilon^{3 / 2} t
$$

where $C_{0}$ is the phase velocity of the eastward wave. The procedure consists in rewriting (2) using $\zeta$ and $\tau$ and then seeks a solution in a power series expansion in the amplitude parameter $\epsilon$. Then by collecting terms of order $\mathrm{O}\left(\epsilon^{3 / 2}\right)$, we obtain the following linear equation in $\psi_{1}$ :

$$
\left(U-C_{0}\right) \frac{\partial}{\partial \zeta}\left(\frac{\partial^{2} \psi_{1}}{\partial y^{2}}\right)+\left[\beta-\frac{d^{2} U}{d y^{2}}\right] \frac{\partial \psi_{1}}{\partial \zeta}=0 .
$$

Also the terms of order $\mathrm{O}\left(\epsilon^{5 / 2}\right)$ give:

$$
\begin{aligned}
& \frac{\partial}{\partial \tau}\left(\frac{\partial^{2} \psi_{1}}{\partial y^{2}}\right)+\left(U-C_{0}\right) \frac{\partial^{3} \psi_{1}}{\partial \zeta^{3}} \\
& \quad+\frac{\partial \psi_{1}}{\partial \zeta} \frac{\partial^{3} \psi_{1}}{\partial y^{3}}-\frac{\partial \psi_{1}}{\partial y} \frac{\partial}{\partial \zeta}\left(\frac{\partial^{2} \psi_{1}}{\partial y^{2}}\right)=0
\end{aligned}
$$

Solutions of (6) and (7) are sought in the amplitude $A(\zeta, \tau)$ and modulated by the meridional function $\varphi(y)$, as given by the relation

$$
\Psi(\zeta, y, \tau)=A(\zeta, \tau) \varphi(y)
$$


(a) Determination of $\varphi(y)$. By substituting relation (8) into

(6), we set the following eigenvalue equation for $\varphi(y)$ :

$$
\varphi^{\prime \prime}(y)+\frac{\beta-U^{\prime \prime}}{U-C_{0}} \varphi(y)=0,
$$

where the prime denotes differentiation with respect to $y$. The boundary conditions are the same as those given by relation (3)

$$
\varphi=0 \quad \text { at } y=0, \quad y=L .
$$

Equation (9) is solved numerically by using GAUSS-SEIDEL's relaxation methods. The shape of horizontal shear is chosen such that the wind is zero at the boundaries. Using centreddifference differentiation, (9) is rewritten as

$$
\varphi_{i}=\frac{\left[\varphi_{i+1}+\varphi_{i-1}\right]}{\left[2-F_{i}(\Delta y)^{2}\right]},
$$

where $\Delta y$ is the grid size, $i=1,2, \ldots, N$, and $F_{i}=$ $\left[\left(\beta-U^{\prime \prime}\right) /\left(U-C_{0}\right)\right]_{i}$.

(b) Nonlinear Waves. By substituting relation (8) into (7), we can obtain a nonlinear equation as the $\mathrm{KdV}$ equation in the form

$$
\frac{\partial A}{\partial \tau}+a_{n} A \frac{\partial A}{\partial \zeta}+b_{n} \frac{\partial^{3} A}{\partial \zeta^{3}}=0
$$

where parameters $a_{n}$ and $b_{n}$ are determined by eigenfunctions $\varphi$ and depend on the profile of $U(y)$ (see Appendix A for more details). They are given by the following expression:

$$
\begin{gathered}
a_{n}=\frac{\int_{0}^{L}\left[\varphi \varphi^{\prime \prime \prime}-\varphi^{\prime} \varphi^{\prime \prime}\right] d y}{\int_{0}^{L} \varphi^{\prime \prime} d y}, \\
b_{n}=\frac{\int_{0}^{L}\left(U-C_{0}\right) \varphi d y}{\int_{0}^{L} \varphi^{\prime \prime} d y} .
\end{gathered}
$$

We now examine solutions of (12) in the form of nonlinear progressive waves (soliton) $A=A\left(\zeta+C_{1} \tau\right)$, where $C_{1}$ is the phase velocity of the soliton which is a weak contribution to the principal phase velocity $C_{0}$. Thus, the total velocity of the system is

$$
C=C_{0}+\epsilon C_{1}
$$

The solitary waves, solution of (12) is given by the following relation (see Appendix B for more details):

$$
A(\zeta, \tau)=A_{0} \operatorname{Sech}^{2}\left[\kappa\left(\zeta-C_{1} \tau\right)\right],
$$

where $A_{0}=3 C_{1} / a_{n}$ is the nonlinear wave amplitude and $\kappa=$ $(1 / 2)\left(C_{1} / b_{n}\right)^{1 / 2}$. Going back to the original variable, we finally have

$$
A(x, t)=A_{0} \operatorname{Sech}^{2}\left(\frac{x-C t}{\Delta}\right)
$$

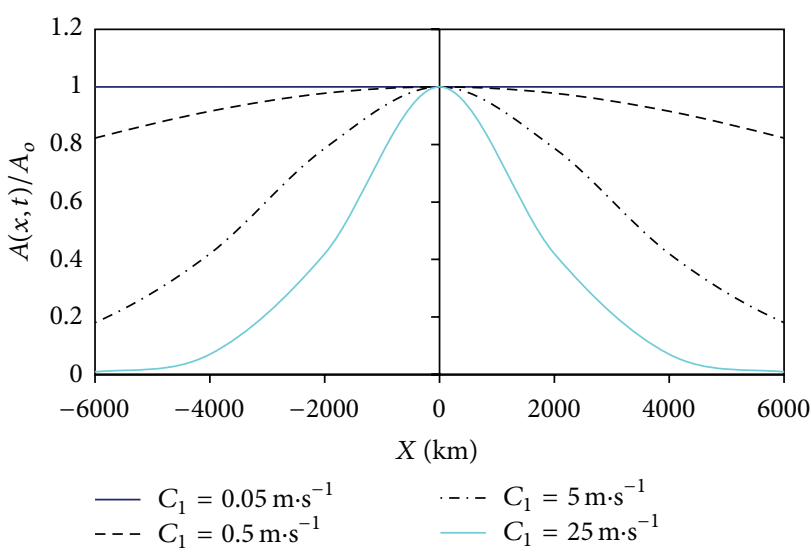

FIGURE 1: Variations of $A(x, t) / A_{0}$ in the propagation direction for different values of the additional wind velocity $C_{1}$.

where $\Delta=\sqrt{b_{n} / \epsilon C_{1}}$ is the soliton characteristic width.

The product of the soliton amplitude and the square of its characteristic width are independent of the soliton phase velocity $C_{1}$. It is however proportional to $b_{n}$ and inversely proportional to $a_{n}$ or $\epsilon$ and is expressed as

$$
\Delta^{2} A_{0}=\frac{3 b_{n}}{\epsilon a_{n}} \text {. }
$$

Since $a_{n}$ and $b_{n}$ are given by relation (13) and (14), respectively, the only known remaining is $\epsilon$. Figure 1 shows some profiles of $A(x, t) / A_{0}$ for different values of additional phase velocity $C_{1}$ and $\epsilon=0.01$. For small $C_{1}$, the figure shows that $A(x, t)$ is nearly constant in space but takes the form of a pulse when this parameter becomes important. It is to be noticed that this parameter appears explicitly in the expression of $\Delta$. Thus, when $C_{1}$ is set to zero, $\Delta$ is even larger and the wave becomes evanescent. But as $C_{1}$ increases, for example, $C_{1}=25 \mathrm{~m} \cdot \mathrm{s}^{-1}$, the wave propagates symmetrically below the plan which passed trough the origin where $x=C t$. Hence, we can say that the wave amplitude $A_{0}$ grows with the additional phase velocity $C_{1}$ (Figure 2 ). This shows that the wave moves faster as its amplitude becomes larger. Figure 2 also shows that the soliton characteristic width $\Delta$ decreases with $C_{1}$.

2.3. Data. During the African Monsoon Multidisciplinary Analysis (AMMA) SOP3 experimentation period, observations were conducted over West Africa from August 15 to September 15, 2006 [20]. The climatology data are from AMMA SOP3 operational database. From the briefing made during this campaign, the convective activity was very high and data for the entire West African region was available from August 15 to September 15, 2006, when wave 2 on the 7 waves was observed over this period. For more details about the 7 AEWs observed during this period, see the paper of [20]. Wave 2 was initiated during the period August 20 to 24, 2006. The satellite data used for this study are digitized images from Meteosat 8 calibrated from the MSG channel IR10.8, representing equivalent blackbody temperatures emitted by clouds. Apart from snow and ice artefacts, contiguous areas 


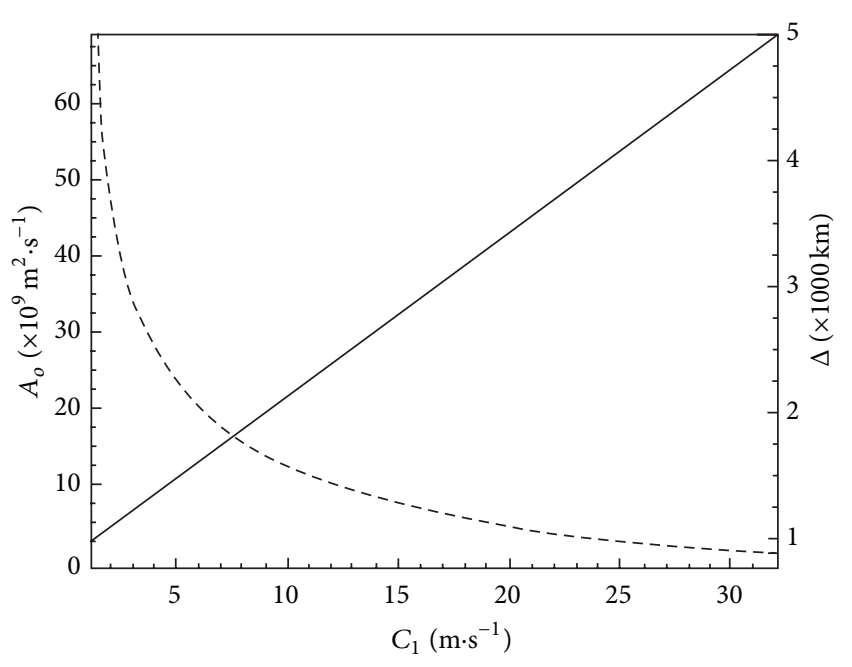

FIgUre 2: Variations of amplitude $A_{0}$ (in solid line) and the width $\Delta$ (in dash line) of the soliton as function of the additional wind velocity $C_{1}$.

of depressed brightness temperatures in $85 \mathrm{GHz}$ imagery indicate the presence of deep convection. The magnitudes of the brightness temperatures are inversely correlated with the strength of the convective updraughts producing the ice hydrometeors. Unlike infrared imagery, $85 \mathrm{GHz}$ imagery can be used to analyse quantitatively the intensity of deep convection. This allows it to be used to study the properties of convective systems at a variety of spatial scales.

\section{Results and Discussions}

As we know, the solitary wave can be considered only when we define a coordinates system which moves with the wave at the velocity $C$, for the soliton to appear stationary. At the origin $X=0$, where $X=x-C t$, the amplitude solitary wave is maximal. This is due to the fact that in the translation $X=x-$ $C t$ of a nonfixed distance $C t$, the maximum wave amplitude, initially at $x=0$, stays until we are at $X=0$.

Explicitly, the streamline depends on the soliton phase velocity $C_{1}$ and on the form of the mean zonal wind $U(y)$. We adopt in this work a basic flow with a horizontal shear as proposed by [21] to describe the midtropospheric jet in the West African Tropical zone. Based on observations the zonal wind can readily be represented in the functional form

$$
U(y)=-U_{0} \sin ^{2}\left(\frac{\pi}{L} y\right)
$$

where $U_{0}=20 \mathrm{~m} / \mathrm{s}$ is the maximal value of wind at the centre of jet $\left(15^{\circ} \mathrm{N}\right)$ and $L$ the distance between the equator $(y=0)$ and $30^{\circ} \mathrm{N}$ latitude $(y=L)$. This jet corresponds to the one seen in the atmosphere during summer at an altitude of around $3000 \mathrm{~m}$ in the Northern African troposphere. The principal phase velocity is found to be $7.0 \mathrm{~m} / \mathrm{s}$ [22-24].

Before examining the influence of the additional wind velocity $C_{1}$ in the present theory, let us first consider the case of linear waves.

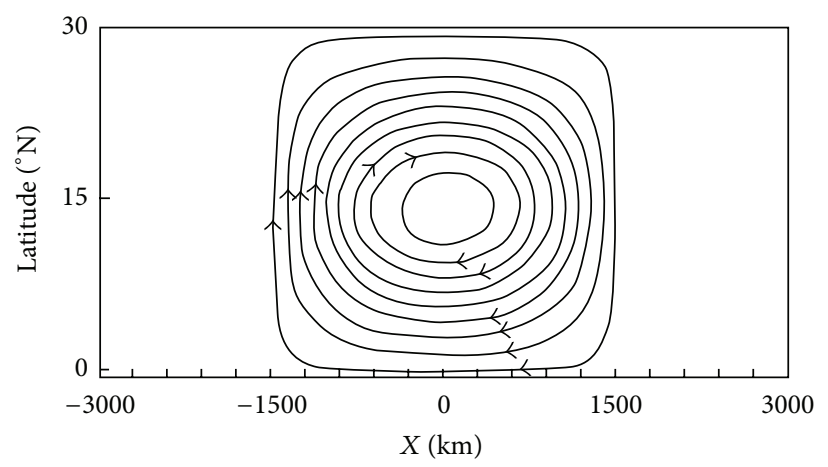

FIGURE 3: Configuration of streamlines in the case of linear approximation with $C_{0}=10 \mathrm{~m} \cdot \mathrm{s}^{-1}$.

3.1. Analysis of Linear Effects. In the linear theory case, the solution of (2) without the Jacobian term is sought in the normal mode:

$$
\Psi(x, y, t)=Y(y) \exp [i k(x-C t)],
$$

where $k$ is the zonal wavenumber, $k=2 \pi / \lambda, \lambda$ the zonal wavelength, and $Y(y)$ is the amplitude function which depends only on $y$ and solution to the following equation:

$$
Y^{\prime \prime}+\left[\frac{\beta-U^{\prime \prime}}{U-C_{0}}-k^{2}\right] Y=0
$$

This equation differs from (9) by the presence of the $k^{2}$ term, but it still must verify the boundary conditions $Y=0$ at $y=0$ and $y=L$. The numerical solution of (21) is found as earlier by GAUSS-SEIDEL's relaxation methods.

Figure 3 illustrates the configurations of streamlines in the $(X, y)$ plane obtained from this approximation. We note that the region of instability corresponds to the depression centred along the principal axes of the jet. These streamlines have a quasi-concentric form, on the one hand symmetric respect of the plan passing $X=0$ and on the other hand to the jet axis.

3.2. Nonlinear Effects. The streamlines in the case of the weakly nonlinear approximation are presented in Figure 4 for different values of the additional wind velocity $C_{1}$. We see that this velocity has a predominant role in the configuration of patterns in the domain under consideration.

For $C_{1}=0.05 \mathrm{~m} \cdot \mathrm{s}^{-1}$ (Figure 4(a)), the patterns are essentially parallel to the zonal direction. Here, the perturbations are swamped by the mean flow and this explains why for weak value of $C_{1}$, one cannot observe the track of the wave. The air flow can be assimilated in this case to the displacement of a solid that presents an axis of symmetric.

When the value of the parameter $C_{1}$ is increased, the streamlines have a new configuration as shown in Figure 4(b). We can deduce that the streamlines tend to unclose and stretch in the zonal direction isolating a depression centred at the region of maximum shear. The presence of a depression characterises the linear effects in the system. The fact that it is presently limited to the maximum disturbance region 


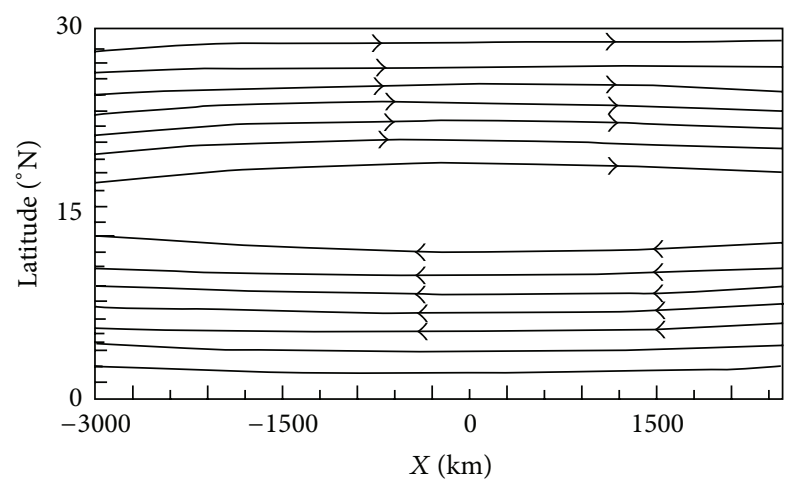

(a)

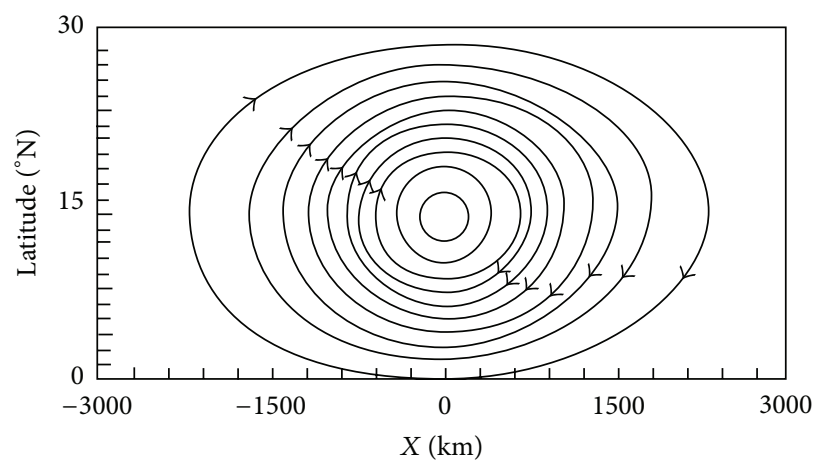

(c)

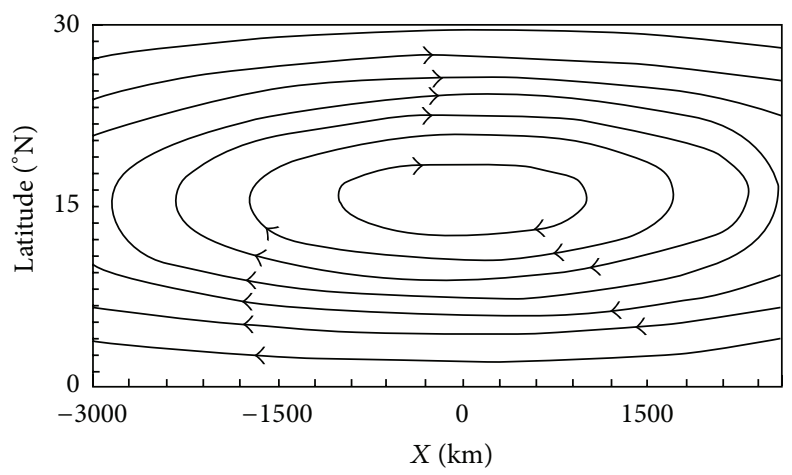

(b)

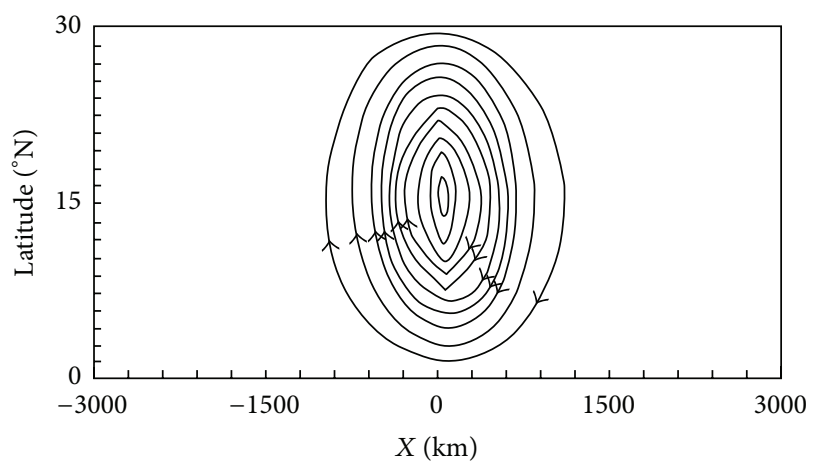

(d)

Figure 4: Configuration of streamlines in the case of weakly nonlinear approximation with $C_{0}=10 \mathrm{~m} \cdot \mathrm{s}^{-1}$ and $\epsilon=0.01$ for (a) $C_{1}=0.05 \mathrm{~m} \cdot \mathrm{s}^{-1}$; (b) $C_{1}=0.5 \mathrm{~m} \cdot \mathrm{s}^{-1}$; (c) $C_{1}=5 \mathrm{~m} \cdot \mathrm{s}^{-1}$; and (d) $C_{1}=25 \mathrm{~m} \cdot \mathrm{s}^{-1}$.

shows that these effects are in their early stages. This is why little is felt at the boundary of the domain, where only weak deformations of streamlines are observed.

We can continue to increase the value of the phase velocity of the soliton in order to determine a value that for which the weakly nonlinear theory leads to the same structure of linear waves as given by normal mode theory. Figure $4(\mathrm{c})$ presents the streamlines for $C_{1}=5 \mathrm{~m} \cdot \mathrm{s}^{-1}$. We note that all the patterns have concentric ovoidal form around the region of maximal instability region where the amplitude of the nonlinear wave is high. The difference from previous configurations is the fact that these patterns are zonally limited at $X= \pm 2100 \mathrm{~km}$. Since our interest is to study the behaviour of wave around the jet, this result is not in contradiction with those obtained in the case of the linear approximation but matter confirms that the maximum instability of the jet is located in depressionary area. As also shown in Figure 4(c), this streamline can be superposed to those given by linear approximation (Figure 3). Hence the nonlinear wave is strongly governed by its linear effects. The first manifestation of the nonlinearity effects appears here, as noted by [25]; the presence of a weak nonlinearity in the system can produce important effects capable of countering those due to the dispersion. According to the weakly nonlinear approximation, the soliton results from a balance between linear and nonlinear effects. In other term, it is for the value of $C_{1}$ equal to $5 \mathrm{~m} \cdot \mathrm{s}^{-1}$ that the Rossby soliton may be observed. Its profile described by relation (17) is represented in Figure 1.
As the parameter $C_{1}$ increases, the nonlinear effects grow and the wave patterns are concentrated around the region where their amplitudes grow (Figure $4(\mathrm{~d})$ ). Here, the streamlines tend to stretch along the meridional direction while being confined in a small zonal domain. This shows that the nonlinear waves became strongly localised.

3.3. Case of TS Debby. For energy consideration, we admit the principle that the energy of the perturbation is proportional to the square of amplitude of the wave. So, for weak values of $C_{1}$, the energy of the wave is dissipated in the space $x-C t$. We found that the energy spreads in the space $x-C t$ but in a reasonable interval compared to the purely linear case. However, Figure 4(c) shows that nonlinearity, though weak, leads to live for the perturbation. The value of $C_{1}$ can be found in a mosaic of satellite images for the period August 20-24, 2006, taken every six hours as shown in Figure 5. The total velocity of the wave is around $15 \mathrm{~m} / \mathrm{s}$ where the classical value of AEW velocity is $10 \mathrm{~m} / \mathrm{s}[21,22,24]$; then we deduce that the additional wave (soliton) velocity is about $5 \mathrm{~m} / \mathrm{s}$.

This figure presents a very distinct signal that can be tracked from an initial region of convection, developing through several stages and moving off the African coast. The convection is more active at 0000 and 1800 UTC showing diurnal cycle of deep convection. The diurnal cycle of convection is important in many aspects of climate studies, in particular, through its strong modulation of the radiative budget and precipitation and its control of surface temperature [26]. Diurnal changes in deep convective clouds 

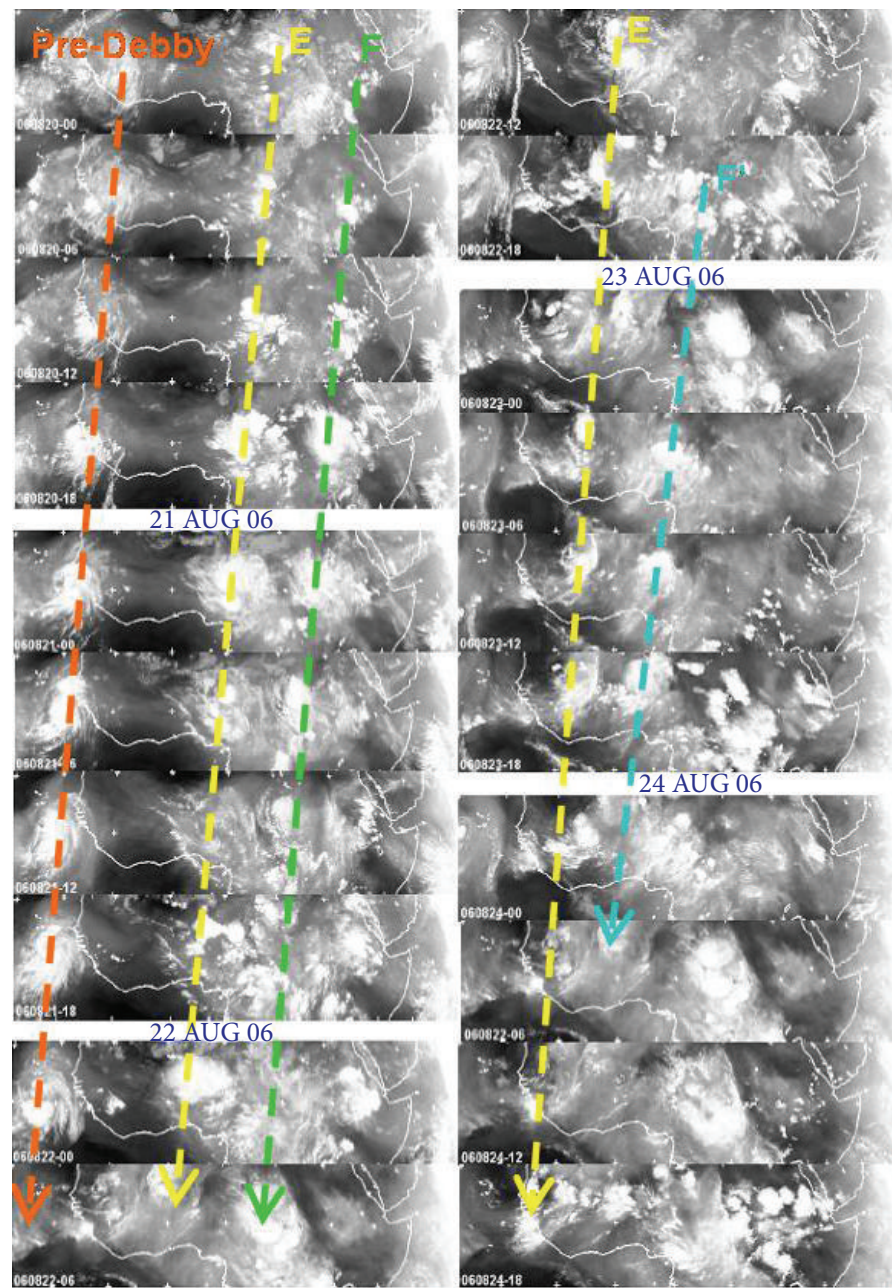

FIgURE 5: The situation on August 20-24, 2006, over West Africa corresponds first to the AEW ridge between the two troughs associated with "pre-Debby" and "F" (August 2100 UTC to August 2206 UTC) with weak convective development over east Guinea, then to the slow and rather inefficient propagation of AEW trough "E" over West Africa. There is no indication of triggering of an AEW (which can be traced back further east) by convection, nor strong development of convection induced by the propagating AEW "F" trough (except for the small thunderstorms on 22nd evening ahead of "E" and the short-lived MCS associated with "E").

influence incoming short wave and outgoing longwave radiations thereby affecting the Earth's radiation balance [27]. Two initial convection cells occur around 0000 UTC on August 21, 2006: the first at the west of the Senegalese coast and the second covers the northeast of Nigeria, south of Niger, and near the Jos Mountain around $15^{\circ} \mathrm{N}$ where the AEW is located. This continental region is favourable for the initiation of long-lived mesoscale convective weather systems which is a manifestation of nonlinear disturbance [28].

Interestingly, this convection begins at a time of the day when the mean MCS genesis frequency over Africa has been shown to be at a minimum [28]. On these images, the brightness of the pixels indicates that the initial convection is located east of and earlier than the first location of the AEW2 (around $20^{\circ} \mathrm{W}$ ). This suggests that convection acts as a precursor for the AEW that develops downstream. Subsequent satellite images show that a convective zone rapidly develops, with high clouds covering a region of $1000 \mathrm{~km}$ square at 0600 UTC on August 21. The brightness of the pixels indicates how the amount of very deep convection begins to propagate westward, crossing Senegal and reaching the eastern Atlantic at approximately 0600 UTC. The "preDebby" cyclonic part of the AEW is over West Africa and propagates westward over the warm waters off the coast of Guinea then becomes TS Debby on August 22, 2006. The cell at the west of the Senegalese coast persists until August 23,2006 , at 0600 UTC. The average speed of the cold cloud $(<190 \mathrm{~K})$ was about $15 \mathrm{~m} / \mathrm{s}$ as noted earlier.

The convection over Jos Mountain remains whereas a new cell appears over the east of Central African Republic. The deep convection does not decrease within the convective region for the following $6 \mathrm{~h}$ as it moves over the Jos Mountain. Deep convection occurs on the leading edge of the remnants of the first convective burst along the coast of the Gulf of Guinea and over Central Africa Republic on August 21, 2006, at 1800 UTC. This convection persists over the entire West African region until August 23, 2006, at 0000 UTC. During the three days, the deep convection which develops 
over central Nigeria, near the Jos Plateau, is stationary, whereas the deep convection over the Senegalese coast marks the beginning of the second convective growth cycle over the Atlantic Ocean. On August 23, 2006, at 1800 UTC, the deep convection initiated over Central Africa Republic at 0600 UTC moves westward. In addition to this continuously developing region of convection propagating westward, the IR imagery shows another interesting feature: persistent localized convection occurs between $10^{\circ}$ and $15^{\circ} \mathrm{N}$. As shown by [7], a nonlinear evolution of the $\mathrm{KdV}$ equation can describe the interaction of solitary wave propagation in a zonal shear flow as AEJ.

\section{Conclusion}

We have presented a nonlinear theory to study the evolution of perturbation due to the shear mean wind in the midtropospheric African jet. Its formulation is necessarily complicated, but we have carefully described all the stages which permit to obtain the final result, so that one can use, in some conditions, these results which seem applicable to description of Rossby solitary waves. This requires the choice of additional wind speed $C_{1}$. The use of the Gardner and Morikawa transformations helps us to introduce the phase velocity of the soliton $C_{1}$. Its influence on the structure and the amplitude for streamline is important. Hence, the weakly nonlinear approximation through the KdV theory explains how the solitary Rossby waves are propagated over West Africa. The weakly nonlinear theory leads to explain the evolution of AEW2 which becomes TS Debby on August 22, 2006, when the soliton velocity was $C_{1}=5 \mathrm{~m} \cdot \mathrm{s}^{-1}$. The presence of a weak nonlinearity in the system can produce important effects capable of countering those due to the dispersion.

In the tropical zone, the cyclones are some time presented as the soliton. It is interesting to confront this theory with other observations since the nonlinear amplitude function $A(x, t)$, which is proportional to pressure, presents a maximum when the eastern wave propagation arrives at $X=0$. These results have to be compared to other weakly nonlinear theory models, for example, with model of solitary pancakes Rossby vortices.

\section{Appendices}

\section{A. Determination of Parameters $a_{n}$ and $b_{n}$}

By substituting relation (8) in (7), we obtain

$$
\varphi^{\prime \prime} \frac{\partial A}{\partial \tau}+\left(U-C_{0}\right) \varphi \frac{\partial^{3} A}{\partial \zeta^{3}}+\varphi \frac{\partial A}{\partial \zeta} A \varphi^{\prime \prime \prime}-\varphi^{\prime} A \varphi^{\prime \prime} \frac{\partial A}{\partial \zeta}=0
$$

or

$$
\varphi^{\prime \prime} \frac{\partial A}{\partial \tau}+\left(U-C_{0}\right) \varphi \frac{\partial^{3} A}{\partial \zeta^{3}}+\left(\varphi \varphi^{\prime \prime \prime}-\varphi^{\prime} \varphi^{\prime \prime}\right) A \frac{\partial A}{\partial \zeta}=0
$$

and if we integrate this equation into meridional domain, we have

$$
\begin{gathered}
\int_{0}^{L} \varphi^{\prime \prime} \frac{\partial A}{\partial \tau} d y+\int_{0}^{L}\left(U-C_{0}\right) \varphi \frac{\partial^{3} A}{\partial \zeta^{3}} d y \\
+\int_{0}^{L}\left(\varphi \varphi^{\prime \prime \prime}-\varphi^{\prime} \varphi^{\prime \prime}\right) A \frac{\partial A}{\partial \zeta} d y=0 .
\end{gathered}
$$

By setting $\int_{0}^{L} \varphi^{\prime \prime} d y$, (A.3) can be rewritten at last as follows:

$$
\frac{\partial A}{\partial \tau}+a_{n} A \frac{\partial A}{\partial \zeta}+b_{n} \frac{\partial^{3} A}{\partial \zeta^{3}}=0
$$

with

$$
\begin{gathered}
a_{n}=\frac{\int_{0}^{L}\left[\varphi \varphi^{\prime \prime \prime}-\varphi^{\prime} \varphi^{\prime \prime}\right] d y}{\int_{0}^{L} \varphi^{\prime \prime} d y}, \\
b_{n}=\frac{\int_{0}^{L}\left(U-C_{0}\right) \varphi d y}{\int_{0}^{L} \varphi^{\prime \prime} d y} .
\end{gathered}
$$

\section{B. Solution of the KdV-Equation}

To solve (12), we introduce the following Galilee transformation:

$$
s=\zeta-C_{1} \tau
$$

In this new referential where the wave is propagated with the velocity $C_{1}$, (12) becomes

$$
-C_{1} \frac{\partial A}{\partial s}+a_{n} A \frac{\partial A}{\partial s}+b_{n} \frac{\partial^{3} A}{\partial s^{3}}=0 .
$$

The integration of (B.2) with respect to $s$ gives

$$
-C_{1} A+a_{n} \frac{A^{2}}{2}+b_{n} \frac{\partial^{2} A}{\partial s^{2}}=0
$$

By multiplying this last equation by $d A / d s$ and integrating them, we have

$$
-C_{1} \frac{A^{2}}{2}+a_{n} \frac{A^{3}}{6}+\frac{b_{n}}{2}\left(\frac{d A}{d s}\right)^{2}=0
$$

or

$$
\frac{d A}{d s}=\left(\frac{C_{1}}{b_{n}}\right)^{1 / 2} A \sqrt{1-\frac{a_{n}}{3 C_{1}} A}
$$

By setting $\widetilde{A}=a_{n} A /\left(3 C_{1}\right)$, we obtain the integral

$$
\int \frac{d \widetilde{A}}{\widetilde{A}(1-\widetilde{A})^{1 / 2}}=\left(\frac{C_{1}}{b_{n}}\right)^{1 / 2}\left(s-s_{0}\right) .
$$

If we assume $s_{0}=0$, (B.6) can be written as follows:

$$
\operatorname{Arg} \operatorname{Sech} \widetilde{A}=\left(\frac{C_{1}}{b_{n}}\right)^{1 / 2} s
$$

And at last

$$
A=\frac{3 C_{1}}{a_{n}} \operatorname{Sech}^{2}\left[\left(\frac{C_{1}}{b_{n}}\right)^{1 / 2} s\right] .
$$




\section{Acknowledgment}

This research is supported by ICTP, Trieste, Italy, through the Associate and Federation Schemes Program (Reference 431).

\section{References}

[1] J. R. Holton, An Introduction to Dynamic Meteorology, vol. 16 of International Geophysics Series, 4th edition, 2004.

[2] T. A. Guinn and W. H. Schubert, "Hurricane spiral bands," Journal of the Atmospheric Sciences, vol. 50, no. 20, pp. 33803403, 1993.

[3] A. Lenouo, F. N. Kamga, and E. Yepdjuo, "Weak interaction in the African easterly jet," Annales Geophysicae, vol. 23, no. 5, pp. 1637-1643, 2005.

[4] R. R. Long, "Solitary waves in the westerlies," Journal of the Atmospheric Sciences, vol. 21, no. 2, pp. 197-200, 1964.

[5] L. H. Larsen, "Comments on solitary waves in the westerlies," Journal of the Atmospheric Sciences, vol. 22, no. 2, pp. 222-224, 1965.

[6] D. J. Benney, "Long nonlinear waves in fluid flows," Journal of Mathematical Physics, vol. 45, pp. 52-63, 1966.

[7] L. G. Redekopp and P. D. Weidman, "Solitary Rossby waves in zonal shear flows and their interactions," Journal of the Atmospheric Sciences, vol. 35, pp. 790-804, 1978.

[8] J. W. Miles, "On solitary Rossby waves," Journal of the Atmospheric Sciences, vol. 36, no. 7, pp. 1236-1238, 1979.

[9] B. J. Hoskins and T. Ambrizzi, "Rossby wave propagation on a realistic longitudinally varying flow," Journal of the Atmospheric Sciences, vol. 50, no. 12, pp. 1661-1671, 1993.

[10] D. Luo, "A barotropic envelope Rossby soliton model for blockeddy interaction. Part II: role of westward-travelling planetary waves," Journal of the Atmospheric Sciences, vol. 62, no. 1, pp. 22 40, 2005.

[11] M. Tepper, "A proposed mechanism of squall line. The pressure jump line," Journal of Meteorology, vol. 7, no. 1, pp. 21-29, 1950.

[12] A. J. Abdullah, "The atmospheric solitary wave," Bulletin of the American Meteorological Society, vol. 10, pp. 511-518, 1955.

[13] E. M. Dobryshman, "Theoretical studies of tropical waves," GATE, vol. 25, pp. 121-177, 1982.

[14] H. S. Huang Sixun and Z. Ming, "Periodic, solitary and discontinuous periodic solution of nonlinear waves in the atmosphere and their existence. Part I and II.," Scientia Sinica B, vol. 31, no. 12, pp. 1489-1502, 1988.

[15] J. W. Rottman and F. Einaudi, "Solitary waves in the atmosphere," Journal of the Atmospheric Sciences, vol. 50, no. 14, pp. 2116-2136, 1993.

[16] Y. L. Yuh-Lang Lin and R. C. Goff, "A study of a mesoscale solitary wave in the atmosphere originating near a region of deep convection," Journal of the Atmospheric Sciences, vol. 45, no. 2, pp. 194-205, 1988.

[17] R. K. Smith and B. R. Morton, "An observational study of northeasterly "morning glory" wind surges," Australian Meteorological Magazine, vol. 32, pp. 155-175, 1984.

[18] T. D. Kaladze, "New solution for nonlinear pancake solitary Rossby vortices," Physics Letters A, vol. 270, no. 1-2, pp. 93-95, 2000.

[19] Q. Li and D. M. Farmer, "The generation and evolution of nonlinear internal waves in the deep basin of the South China Sea," Journal of Physical Oceanography, vol. 41, no. 7, pp. 13451363, 2011.
[20] E. J. Zipser and Coauthors, "The Saharan air layer and the fate of African easterly waves: the NAMMA field program," Bulletin of the American Meteorological Society, vol. 90, pp. 1137-1156, 2009.

[21] M. A. Rennick, "The generation of African wave," Journal of the Atmospheric Sciences, vol. 33, no. 10, pp. 1955-1969, 1976.

[22] R. W. Burpee, "The origin and structure of easterly waves in the lower troposphere of North Africa," Journal of the Atmospheric Sciences, vol. 29, no. 1, pp. 77-90, 1972.

[23] C. Mass, "A linear equation model of African wave disturbances," Journal of the Atmospheric Sciences, vol. 36, pp. 20752092, 1979.

[24] A. Lenouo and F. Mkankam Kamga, "Sensitivity of African easterly waves to boundary layer conditions," Annales Geophysicae, vol. 26, no. 6, pp. 1355-1363, 2008.

[25] B. B. Kadomtsev, Phénomènes Collectifs dans le Plasmas, Editions Mir, Moscou, Russia, 1979.

[26] R. A. Houze Jr., "Mesoscale convective systems," Reviews of Geophysics, vol. 42, no. 4, 2004.

[27] L. A. T. Machado, H. Laurent, and A. A. Lima, "Diurnal march of the convection observed during TRMM-WETAMC/LBA," Journal of Geophysical Research D, vol. 107, no. 20, pp. 31.1-31.15, 2002.

[28] K. I. Hodges and C. D. Thorncroft, "Distribution and statistics of african mesoscale convective weather systems based on the ISCCP meteosat imagery," Monthly Weather Review, vol. 125, no. 11, pp. 2821-2837, 1997. 

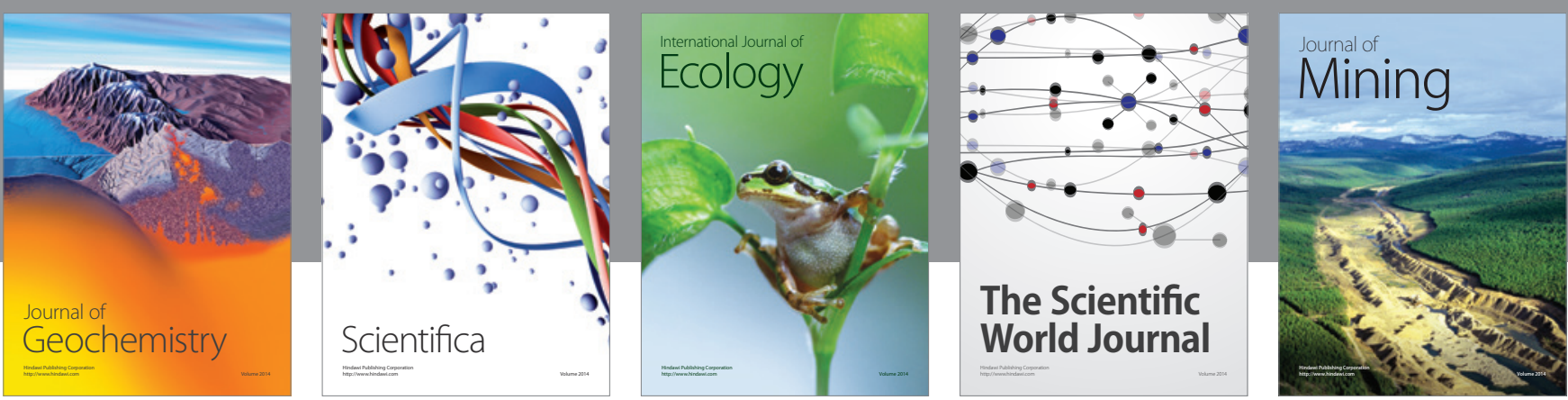

The Scientific World Journal
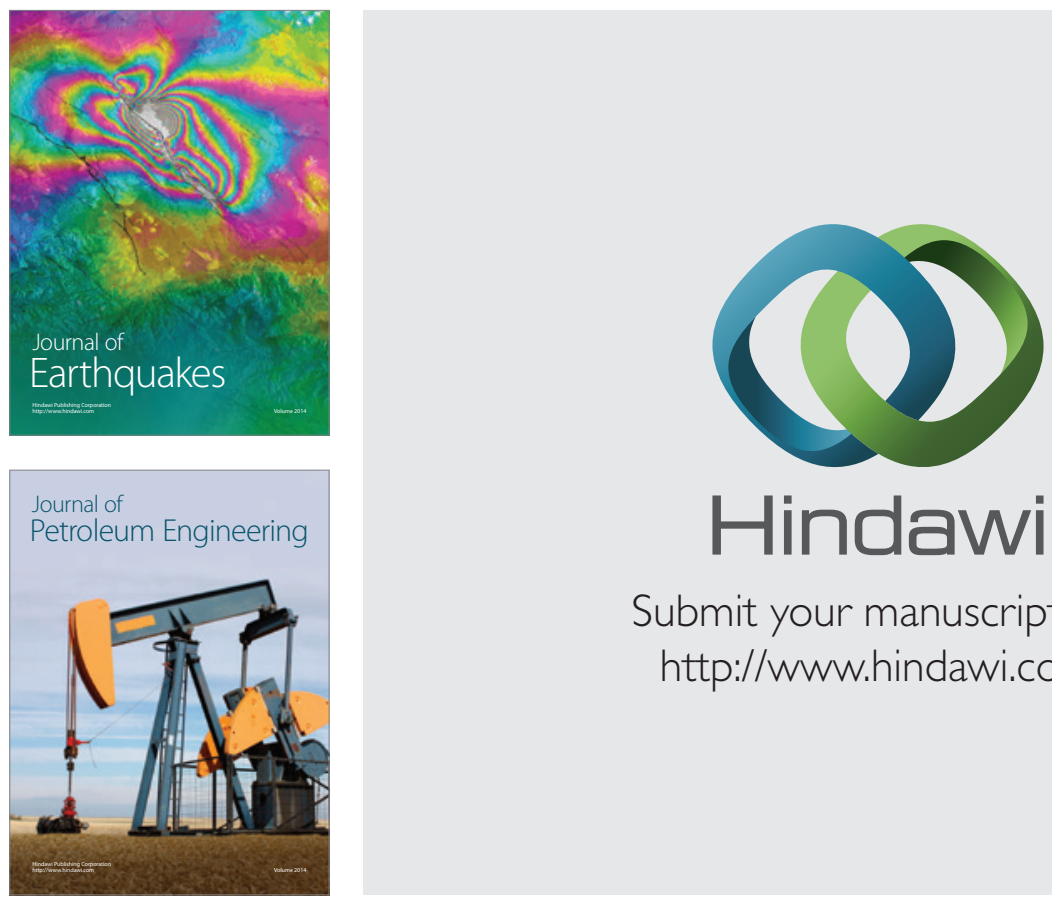

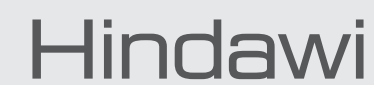

Submit your manuscripts at

http://www.hindawi.com
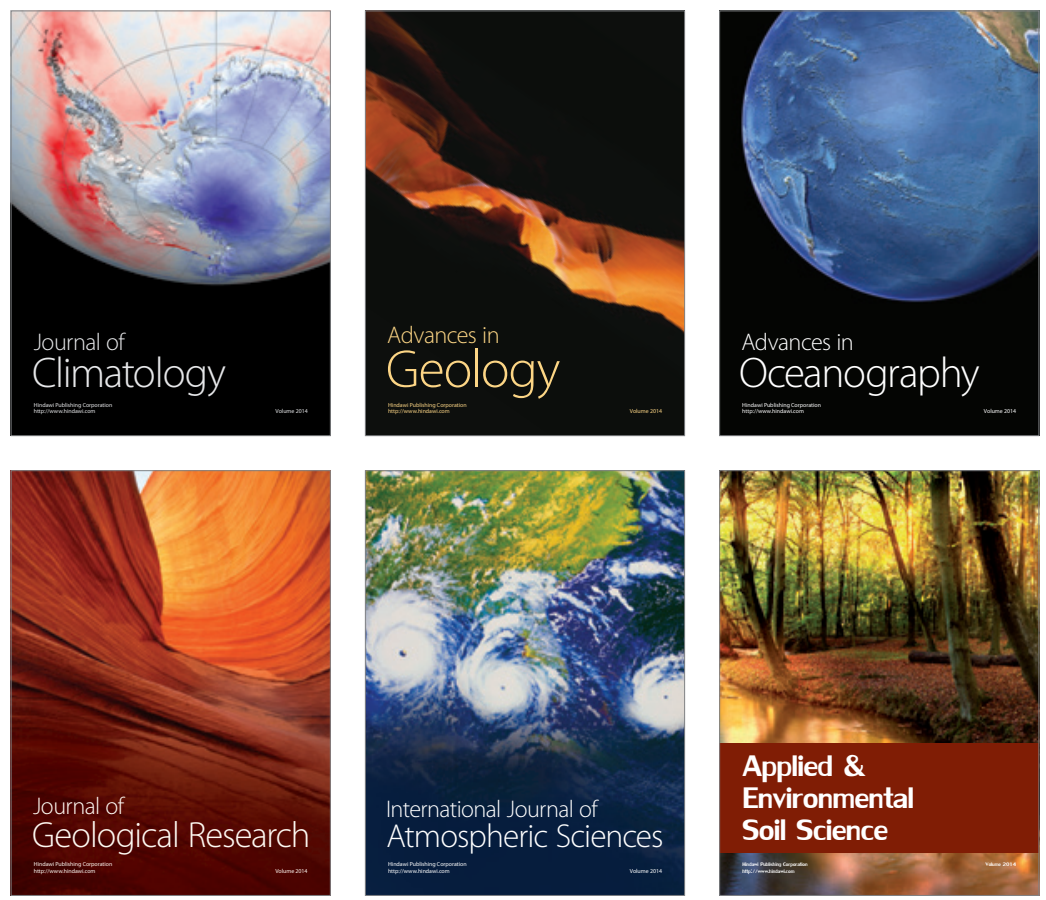
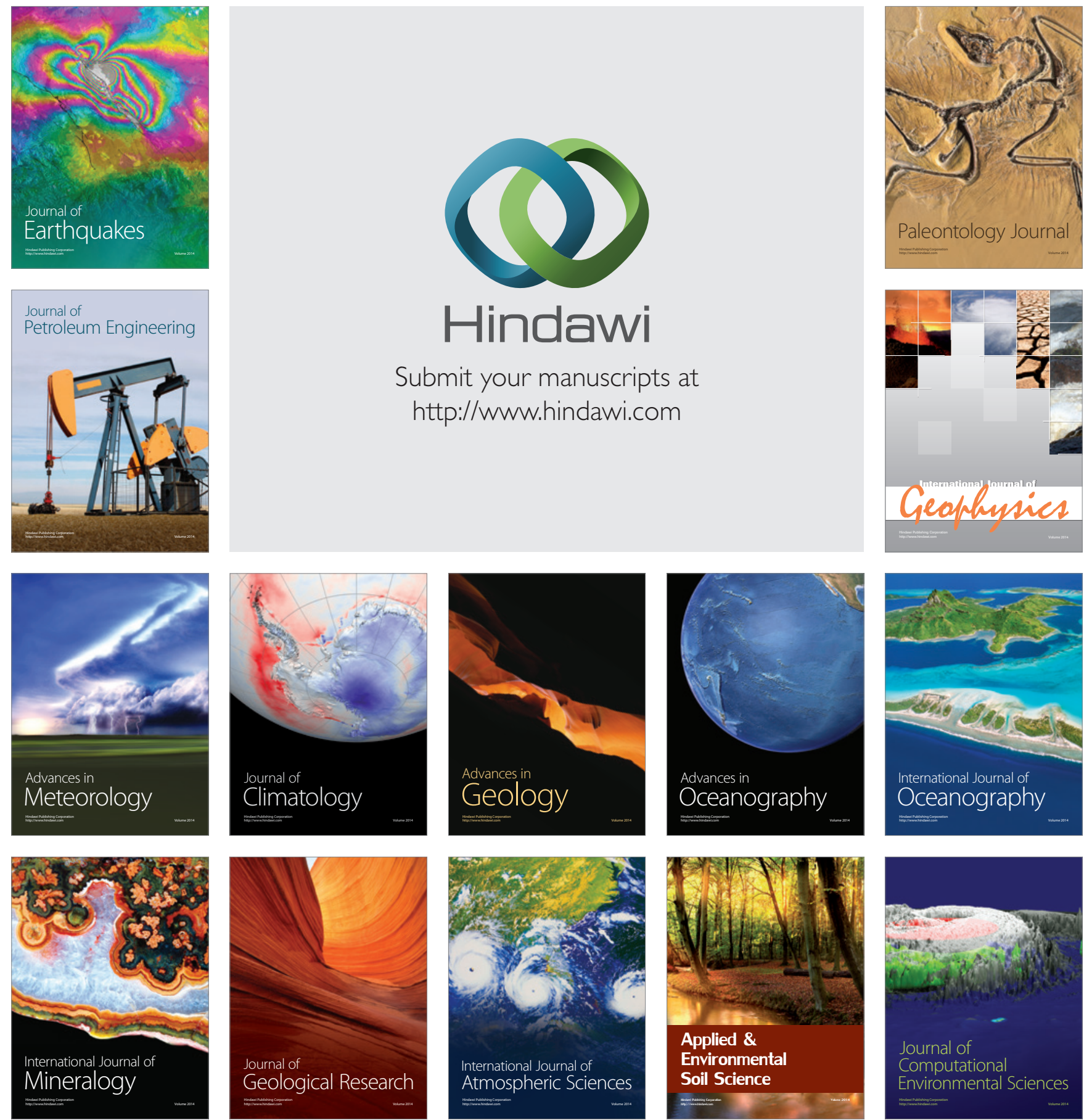